\title{
Filosofía y Enseñanza Jurídica
}

\author{
Philosophy and Legal Teaching
}

Carmela Mollinedo Garay ${ }^{1}$

\section{Resumen}

En el presente trabajo, voy a partir desde las diferentes concepciones de sujeto, de distintos filósofos, como Platón Aristóteles, Rousseau, Pestalozzi, Descartes, Hume, Herbart, Marx y Durkheim.

Una vez presentadas las ideas sobre la concepción de sujeto de los autores, se abordará, su forma de concebir e incidir en la pedagogía, y finalmente relacionar la filosofía de la educación con el sistema implementando en la enseñanza jurídica, que, como actual abogada, ex alumna de la carrera de grado y hoy alumna de posgrado, es uno de mis principales objetos de interés.

Palabras clave: Enseñanza de Derecho, Filosofía, Pedagogía

\section{Abstract}

In the present work, I will start from the different conceptions of people, from different philosophers, such as Plato Aristotle, Rousseau, Pestalozzi, Descartes, Hume, Herbart, Marx and Durkheim. On this occasion.

Once the ideas on the authors' conception of the subject have been presented, their way of conceiving and influencing pedagogy will be addressed, and finally relating the philosophy of education with the system implementing in legal education, which, as a current lawyer, A former undergraduate student and now a graduate student, she is one of my main objects of interest.

Keywords: Teaching of Law, Philosophy, Pedagogy

\section{Definiciones Preliminares}

Enseñanza: Acto de una persona (profesor) que transmite a otra persona (alumno) unos conocimientos sobre una materia determinada.

Educación: Desarrollar la capacidad intelectual, moral y afectiva de las personas de acuerdo con la cultura y las normas de convivencia de la sociedad a la que pertenecen.

Recibido: 12 de marzo de 2021 Aceptado: 25 de junio de 2021 Publicado: 8 de julio de 2021

1 Abogada, Carmela Mollinedo (UNC), Córdoba, Argentina. Magistranda (UNR), Rosario, Argentina. Correo electrónico: mollinedogarayc@gmail.com ID https://orcid.org/0000-0001-5915-3480 
Teoría general de Derecho: Ciencia jurídica que estudia los elementos del derecho u ordenamiento jurídico existente en toda organización social y los fundamentos científicos y filosóficos que lo han permitido evolucionar hasta nuestros días.

Trialismo: Teoría jurídica elaborada por el jurista alemán Werner Goldschmidt. Werner Goldschmidt sostiene que el fenómeno jurídico es una totalidad compleja que denomina "Mundo Jurídico".

Pensamiento crítico: Capacidad de analizar y evaluar razonamientos.

Derechos Inalienables a la persona humana: Aquellos derechos que, de acuerdo a la constitución, y tratados internacionales, se tienen por el simple hecho de ser persona.

\section{Introducción}

Sin dejar de lado, que la educación es un derecho fundamental, e inalienable a la persona humana, el presente trabajo aborda la educación, como verbo, es decir como presentación sistemática de ideas a los y las estudiantes. En otras palabras, se centrará en la educación como ciencia.

La ciencia de la educación es también conocida como pedagogía, cuyo origen es la filosofía. En el rubro, actualmente, se requieren profesores de formación integral, es decir que son necesarios, profesores que sepan tanto del contenido del área que enseña, como de la ciencia de la educación. Esto implica que los profesores deben ser formados a tales fines. (Biswas, 2007)

La pedagogía como ciencia de la educación, se encuentra íntimamente relacionada, con la epistemología, que es, la discusión sobre el conocimiento, esto significa, que hace un estudio preliminar sobre el conocimiento, por lo que podría decirse que es la ciencia de las ciencias.

Ahora bien, antes de estudiar el conocimiento en sí, resulta necesario hacer referencia a las concepciones sobre aquél que conoce, "los sujetos", ¿por qué?, primero porque parece oportuno, antes de hablar de la actividad, hablar de quien la realiza, pero por otro lado, las distintas concepciones que se hayan tenido sobre quienes serán educandos en el presente trabajo, implica sobre ellas características y capacidades diferentes, y por ende la forma en la que responden ante la educación es teóricamente, distinta.

Para dar un ejemplo, en rasgos generales, los empiristas, como David Hume, consideran que el sujeto se constituye en el flujo de percepciones y las impresiones de reflexión (Montero, 2012). Es decir, que aprenden a través de las experiencias.

A diferencia de autores, más bien racionalistas, como René Descartes, que considera que sujetos son quienes crean a través del pensamiento (Campero, 2017), es decir que ven en el educando como algo primordial, la capacidad de razonar, y por lo tanto el aprendizaje se alcanza a través del razonamiento. 
Del mismo modo, autores naturalistas $\mathrm{y} / \mathrm{o}$ constructivistas, tienen distintas percepciones de sujeto, por ende, sobre el modo en que aprenden, y sobre las formas más efectivas de cumplir con la enseñanza.

Una vez analizadas, las formas de educación en razón de las formas de entender al sujeto, lo trasladaré, al campo de la enseñanza jurídica.

A partir de ahí, tendré en cuenta que, la enseñanza del derecho se desarrolla de distinto modo, no solo teniendo en cuenta las características del educando, sino también dependiendo de la percepción que se tenga sobre el derecho.

El Estado Liberal, percibe al derecho como un conjunto de normas y principios, y el Estado Social, lo percibe en razón de su función, siendo ésta la de alcanzar la igualdad real. Esto implica que las distintas escuelas, cumplan con la enseñanza del derecho a través de métodos más teóricos; del método del caso; o del método del caso-problema. ( Carlos ListaAna María Brigido y Silvana Begala, 2007)

\subsection{Diferencias fundamentales en las corrientes}

Los racionalistas, como su nombre lo indica, consideran el razonamiento como un factor central para el conocimiento, porque sostienen que la razón es la fuente más confiable para conocer y que a través de ella se puede conocer la verdad en sí misma, comparando ideas con otras ideas.

Entienden que la "verdad", es aquello que se comparte de forma universal, y consideran que razonando podemos llegar a estos "juicios universales”. (Biswas, 2007)

El empirismo en cambio surge como una reacción frente al racionalismo, consideran, que todo nuestro conocimiento deriva de nuestras experiencias sensoriales, es decir, que solo el conocimiento crea conocimiento y éste sólo proviene de la experiencia. Entienden a la experiencia como el conjunto de dos momentos, la percepción y el reflejo. Siendo la primera el conocimiento de objetos externos y la segunda la internalización de lo percibido. (Biswas, 2007)

Estas dos primeras teorías, tienen en común, que su inquietud se centra en el conocimiento, y es en ello en lo que no se pueden poner de acuerdo, mientras que el naturalismo y el constructivismo se centran en la dirección que debe tomar la educación.

En el Naturalismo, Rousseau, considera que la educación debe estar al desarrollo individual de cada sujeto, y éste a su vez debe ponerse al servicio de la naturaleza del hombre. Este enfoque, es además de naturalista, humanista, porque considera que el educando debe ser el centro, y que es un sujeto individual. Propone salvarlos, de la soledad, el egoísmo y la deshumanización proveniente de la comercialización y la industria. (Campos-Badilla, 2016).

El constructivismo, en términos generales, asume que el conocimiento previo lleva a conocimiento nuevo por medio de procesos de andamiaje. El constructivismo sostiene 
que el aprendizaje es esencialmente activo; es decir que las experiencias se convierten en conocimiento, a través de un proceso de asimilación en base al conocimiento previo. Eso significa, que es un proceso subjetivo. (Campos-Badilla, 2016)

El materialismo histórico se fundamenta en el estudio del desarrollo de las sociedades, determinando cuales son los mecanismos que posibilitan el origen, evolución y muerte de una sociedad, es decir, esta filosofía busca explicar las causas que producen los cambios sociales. Parte de la idea de que la sociedad se organiza en torno a un sistema productivo, y es así como logra entrar en relaciones fijas de producción a través de las cuales se constituye la estructura económica, cimiento sobre el cual se construyen los organismos jurídicos y políticos, a los que corresponde la conciencia social. ${ }^{2}$

El término fue acuñado por Gueorgui Plejánov, marxista que aludía al marco conceptual en que identificaba a $\mathrm{Marx}^{3}$

\section{El Sujeto \\ 3.1 Platón (427 a. C - 347 a.C.)}

Platón, discípulo de Sócrates, concebía al hombre formado por lo material y lo inmaterial, como opuestos, el cuerpo y el alma.

El cuerpo (lo material) es mortal, pero en el mundo sensible es lo que podemos percibir. Sin embargo, esa forma de percepción es incompleta, ya que a través de él no podemos percibir lo que percibiríamos a través del alma, de allí su famosa frase "el cuerpo es la prisión del alma”.

El alma (lo inmaterial), es inmortal, y físicamente inaprensible. El filósofo hace una división tripartita del alma, (lo racional, lo irascible y lo concupiscible)

Lo racional, según Platón, es el medio que les permite a los sujetos conocer, es decir, conocen a través de la razón y de allí proviene el saber, es por ello que ubicamos a Platón entre los racionalistas.

Por otra parte, lo irascible, representa las pasiones del hombre, es su sentido protector. Y por último lo concupiscible, que contiene la función de conservar, conlleva bajos deseos y necesidades físicas, produce templanza. (Larecio, 2010)

"Hombre. Animal sin alas, con dos pies, con las uñas planas; el único entre los seres que es capaz de adquirir una ciencia fundada en razonamientos". (Larecio, 2010)

\subsection{Aristóteles (384 a. C - 322 a. C)}

A diferencia de su maestro (Platón), Aristóteles no divide al hombre, en cuerpo y alma, sino que entiende que el cuerpo y el alma van en conjunto, ya que necesitan el uno del otro.

\footnotetext{
${ }^{2}$ https://conceptodefinicion.de/materialismo-historico/

${ }^{3}$ https://economipedia.com/definiciones/materialismo-historico.html
} 
Pero además de esto, Aristóteles habla del hombre como un "zoon politikon", es decir, un animal político o social, puesto que no puede vivir si no es en comunidad, distinto del resto de los animales y de los Dioses.

Y así como el hombre necesita vivir en comunidad, para lograr la convivencia, las comunidades necesitan de un Estado y de leyes que eduquen a los hombres para la responsabilidad civil. (Aristóteles, como muchos otros, excluye a las mujeres del sistema educativo, y también a los hombres esclavos).

Es un filósofo racionalista al ser discípulo de Platón, piensan que un sistema lógico que parte de verdades, garantiza nuevas verdades y llega al conocimiento verdadero e intuitivo de los principios. Pero Aristóteles en su obra "Metafísica", explica al conocimiento como un proceso.

Este mencionado proceso parte de lo común con los animales dotados de sensación y memoria y, por tanto, con experiencia; es la acumulación de experiencia lo que hace a los seres humanos "expertos".

Más perfecto es el conocimiento de dicha experiencia unida a la reflexión, lo que convierte a los seres humanos, en "artesanos".

La perfección de la función racional humana se manifiesta en la suprema facultad de elevarse a los fundamentos de dichos conocimientos a través de las causas hasta los primeros principios; es en esto en lo que los seres humanos se asemejan a los dioses, el saber de una Ciencia primera, entendida hasta el siglo XVIII como Metafísica. (Larecio, 2010)

\subsection{René Descartes (1596-1650)}

La filosofía de Descartes propone una forma de concebir al mundo estrictamente ligada a la comprensión de la realidad a través del pensamiento.

Descartes, distingue entre el ego cogitans y la res extensa. El origen de la sustancia es Res Cogitans que es una cosa que piensa y no necesita del cuerpo para existir, su atributo es el pensamiento. Es decir que, las sustancias son porque poseen un atributo, una cualidad que las define como ellas mismas y las distinguen unas de otras, por lo tanto, el "yo" existe como una sustancia cuya esencia es pensar y no necesita del cuerpo para existir, su atributo es el pensamiento.

Es decir, distinto de Aristóteles y Platón, para Descartes el alma, es más inteligible que el cuerpo y puede ser lo que es, sin el cuerpo.

Analizando a Descartes, la emergencia del concepto de sujeto se ensambla con la discusión acerca de la idea de verdad, más aún, podemos decir que el conocimiento de la verdad es en Descartes el conocimiento del sujeto

Retomando la idea de que el conocimiento proviene de la mente o de la razón, a partir de tener o poseer un principio verdadero se pueden alcanzar el resto de las verdades 
posibles. En consecuencia, verdad, ciencia y conocimiento, encuentran en la razón un lugar común. Es por esto, que ubicamos a Descartes entre los filósofos racionalistas. (Larecio, 2010)

\subsection{David Hume (1711-1776)}

Hume, observa al hombre y ve dos aristas, el hombre como sujeto creador de conocimiento, y el hombre como objeto de conocimiento.

Es a partir de las cualidades del hombre para conocer, que Hume considera importante la creación de una ciencia del hombre.

"Y como la ciencia del hombre es la única fundamentación sólida de todas las demás, es claro que la única fundamentación sólida que podemos dar a esa misma ciencia deberá estar en la experiencia y en la observación” (Hume, 1984, p. 81)

Como bien evidencia la frase citada, Hume era un filósofo empirista. A diferencia de Descartes ("pienso luego existo"), Hume consideraba que lo real eran las cosas, mientras que las ideas eran meras copias de ellas.

Además, distingue dos elementos del pensamiento, las ideas y las impresiones. La diferencia entre ambas es la intensidad con que las percibimos. Las impresiones son contenidos mentales más intensos y contenidos mentales menos intensos. A su vez, las ideas derivan de las impresiones; las impresiones son, pues, los elementos originarios del conocimiento; de esta relación entre las impresiones y las ideas extraerá Hume el criterio de verdad: "una proposición será verdadera si las ideas que contiene corresponden a alguna impresión; y falsa sino hay tal correspondencia."

Hume advierte que, la capacidad de generar ideas no es ilimitada como parece, sino que provienen de la semejanza, la de continuidad en el tiempo o en el espacio, y la de causa o efecto. Es precisamente esto, lo que lleva a Hume a privilegiar la experiencia y la observación, pues sin ellos, no podemos generar ideas. (Ordosgoitti, 2016)

\section{5 Émile Durkheim (1858-1917)}

Emile Durkheim, además de ser filósofo, era sociólogo, por lo que, desde su perspectiva, el individuo y su subjetividad sólo pueden explicarse luego del estudio de la estructura social que lo constituyen.

El autor presentado, sostiene que la subjetivación del individuo y sus formas de conciencia son el resultado de procesos internos y concomitantes a la estructura social.

A raíz de observar al individuo y su vida en la sociedad desarrolló teorías como la de los “tipos de solidaridad", una es la "solidaridad mecánica", es un fenómeno moral derivado de un determinado tipo de organización societal: el tipo segmentario, que se define por la semejanza o indiferenciación de en un mismo grupo, (semejanza de representaciones: “conciencia colectiva”). La otra forma de solidaridad es la "solidaridad orgánica”: es un tipo 
de cohesión moral que deriva directamente del tipo estructural de diferenciación que le corresponde -el tipo organizado, basado en la diferencia de funciones, o "división del trabajo social"

Sobre la base de todos estos elementos, es posible retomar ahora el estudio de las relaciones y diferencias entre la "conciencia individual" y la "conciencia colectiva" o común.

La sociedad es la que da sentido, la que provee los significados de la vida. Estos significados son los que pueblan y rellenan la interioridad de la conciencia del sujeto. La sociedad, internalizada, es fuente de significados, de sentidos para el sujeto. Él la vive, internamente, como certeza subjetiva, y, externamente, como "realidad". (Funes, 2004)

\subsection{Jean- Jacques Rousseau (1712-1778)}

Al igual que Durkheim, el presente autor se desenvolvía en forma destacada en el ámbito de la sociología. Pero se diferencia en que, mientras Durkheim ve en la sociedad una influencia educadora, Rousseau entiende, que la sociedad, su capitalismo, industria y comercialización corrompen a los sujetos, que en su estado natural crearía y mantendría nuevas reglas y formas socio-políticas. Es decir, que Rousseau confía en la "naturaleza humana".

A partir de lo explicado anteriormente, es que Rousseau sienta uno de los principios fundamentales para la pedagogía actual, y es que, el centro de la educación es el educando, y que la misma debe dirigirse al desarrollo individual y personal de él, para así beneficiar a la sociedad en general (Campos-Badilla, 2016)

\subsection{Johann Heinrich Pestalozzi (1746-1827)}

Pestalozzi, comparte con Rousseau el optimismo por la naturaleza humana, pero se diferencia en que considera que la corrupción es parte de la naturaleza humana, en otras palabras, su postura no es tan pesimista en relación a la sociedad, sino que para este autor "la vida educa".

"Si es la vida la que educa, no vale aislar al educando de la sociedad, puesto que la vida es también experiencia social” (pág. 36, Soto-Bernardini, 2009)

Ésta última cita hace referencia a que, de acuerdo a la postura de Pestalozzi, la educación se debe lograr, atravesando el contexto social del educando, no pudiendo aislar la realidad del sujeto. (Campos-Badilla, 2016)

Esto es lo que nos permite ubicar a Pestalozzi, dentro del constructivismo.

\subsection{Johann Friedrich Herbart (1776-1841)}

Partiendo de las ideas de Pestalozzi, Herbart, entiende que las experiencias del educando deben ser instruidas.

El autor presentado en este punto sostiene que, si bien la voluntad funciona como guía en las acciones de los sujetos, previamente se debe organizar las ideas, se las debe 
instruir. Ya que considera que la información que recibe el educando es lo que construye su conciencia.

Sin embargo, aclara, que no toda instrucción influye educativamente, pues para que así sea, la instrucción debe lograr el interés del educando.

Tras esta última reflexión, el autor pensó en una manera de lograrlo e ideó los pasos formales de la enseñanza. 1) Claridad u ostentación de los objetos. El maestro muestra el objeto para que sea observado en su totalidad. 2) Asociación o comparación. El espíritu une lo que hay de nuevo en lo mostrado con las ideas ya conocidas. 3) Sistematización y generalización. El alumno se encuentra en condiciones de formular nociones abstractas y ordenarlas. 4) Método o aplicación. Los resultados de las etapas anteriores son aplicados en casos concretos (etapa práctica)

Pero el resultado de los pasos formales no fue el esperado, pues al aplicarse en toda enseñanza y en toda materia, se convirtió en una práctica rutinaria y deshumanizada. ${ }^{4}$ (Zuretti, 1998)

\subsection{Karl Marx (1818-1883)}

Lo más destacado en Marx, es que, él lleva la filosofía a la práctica, entiende que los cambios materiales son decisivos como no lo son los cambios espirituales. Es por ello que es considerado un materialista histórico.

Este pensador, considera que el ser humano es un ser material y todo lo que éste hace es una actividad de transformación del mundo material.

Como es sabido, Marx en "El Manifiesto Comunista", divide la sociedad en la que subsume a los individuos, en clases sociales. Una de ellas es el proletariado, que son quienes prestan su fuerza de trabajo a otra clase social, que es la burguesía, quienes tienen el capital.

El proletariado aprende funciones específicas dentro del sistema productivo del capitalismo, pero no tiene conciencia de todo el proceso productivo. Es por ello que pretende crear conciencia al proletariado sobre su propia situación para que este grupo sea capaz de transformarlo. (Campos-Badilla, 2016)

\section{Pedagogía}

El capítulo I, explica, muy brevemente, que la pedagogía es la ciencia de la educación cuyo origen se encuentra en la filosofía. Y en el capítulo II, introdujimos los principales filósofos, y su idea sobre los educandos.

En el presente capítulo, se abordará, la pedagogía desde una perspectiva más amplia, y como los pensadores elegidos influyeron en la pedagogía a lo largo de la historia.

\footnotetext{
${ }^{4}$ https://historiaybiografias.com/pedagogia-cientifica-herbart/
} 


\subsection{Desarrollo}

Etimológicamente hablando, la palabra pedagogía, proviene del latín piado, que significa niño, y agein, que significa guiar o conducir. Es decir, que el origen etimológico de la palabra significa, guiar al niño, aunque podemos asignarle un significado más amplio, hablando de guiar al educando.

La pedagogía, tiene su fin último en instruir mediante la interiorización de valores, que dignifiquen a la sociedad. La filosofía pedagógica, quiere liberar al mundo de las alienaciones. (Paniagua, 2015)

Éste trabajo, utiliza el término pedagogía, en el sentido de ciencia de la enseñanza o de la educación, aunque todavía se discute sobre si la pedagogía es una ciencia o un arte.

Etimológicamente, ciencia, significa en su traducción literal, conocimiento. Pero también puede identificarse, como el deseo natural de los sujetos de conocer para mejorar su estado de vida.

Por otro lado, educación, (educatio- onis), significa instruir mediante acción docente. Aunque, esa es una definición, que puede llevarnos a creer que la instrucción en un acto de un solo lado, pero en realidad, es un proceso de reciprocidad en el dar y recibir conocimiento. (Paniagua, 2015)

Si buscamos a través de la pedagogía, el desarrollo de las capacidades de los educandos hasta alcanzar su máximo nivel de conocimiento, a través de la transmisión de valores, es esencial la integración de la pedagogía y el conocimiento. Para ello, los profesores deben tener conocimiento de la materia que enseñan, y conocimiento pedagógico, para así saber que para enseñar una determinada materia, a determinados educandos, conviene utilizar determinadas estrategias y técnicas pedagógicas. (Biswas, 2007)

Para lograr el máximo nivel de conocimiento de los educandos, tenemos que entender, que, aunque el conocimiento pedagógico y epistemológico es esencial, ninguna de sus teorías es completa para transmitir conocimiento. Sino que, son complementarias unas a otras, por ello la formación docente debe ser integral. (Biswas, 2007)

\subsection{Influencia de los filósofos en la pedagogía}

Platón, dejó varias obras importantes y reconocidas, una de ellas es "La alegoría de las cavernas", con la cual, pretende hacer una metáfora de la vida, y se centra en el conocimiento.

Los atenienses, eran humanistas, por lo tanto, sus disputas en las escuelas eran intelectuales, se buscaba el conocimiento de la verdad, y Platón soñaba con una república democrática, considerando la influencia de la democracia en la educación.

En cuanto al desarrollo educativo se entiende que existen diversos grados de conocimiento, que dependen de la formación cultural. Esto se puede apreciar en la historia de los ciudadanos y como manejan sus vidas en relación al mundo externo a la caverna, es 
la diferencia que plasma en cuanto a quienes vivían afuera de la caverna desde su infancia, aquel que luego de vivir prisionero, sale y se topa con el mundo exterior y reacciona de cierta forma, y quienes nunca han salido, no se movieron y ni siquiera sintieron las cadenas.

Platón pretendía una educación municipal, ya que considerando que los grados de conocimiento, dependen de la formación cultural de cada educando, es necesario evitar las pretensiones totalitarias y para ello debe haber un control, que debe ser lo más próximo posible de la comunidad. En fin, toda enseñanza debería ser pública. (Gadotti, 2003)

En síntesis, Platón era defensor de la educación pública, que tanta importancia tiene aún en el Siglo XXI. Argentina, se destaca por la educación pública universitaria, pero el modelo al que tiende es a la educación pública desde el primer nivel, que ha llevado a países como Finlandia a un reconocido éxito.

Su discípulo, Aristóteles, se distingue en que para él las medidas educacionales son "condicionantes", es decir que creía que el hombre a través de la educación podía convertirse en alguien bueno o en alguien malo. (Gadotti, 2003)

Éste filósofo, dividía la educación en dos, por un lado, estaba la educación moral y por otro la educación intelectual, ambas dos igual de importantes. Además, seguía un plan educativo basado en cinco periodos educativos:

El primero era la infancia, se trataba del periodo de crianza (formación de hábitos), estaba compuesto por "el” juego.

En segundo periodo, alcanzaba hasta los 5 años, consistía en el desarrollo de los buenos hábitos, pero sin lecciones y sin obligaciones. El tercero, la etapa entre los 5 y los 7 años, profundizando en los hábitos.

El cuarto, desde los 7 años hasta la pubertad, era el periodo de educación pública con asignaturas tales como: gimnasia, lectura, escritura, música y dibujo.

Y por último la educación liberal, que se impartía en Liceos, con asignaturas como podrían ser las matemáticas, lógica, metafísica, ética, música, física o biología. La música era considerada como elemento vital en la educación liberal, ya que se consideraba que por una parte contribuía a la formación del carácter y por otra a la purificación emotiva.

Es decir que, define a la educación como un proceso de socialización. Al educarnos somos capaces de asimilar y aprender conocimientos.

Aristóteles, nos enseña que la educación es un proceso de perfeccionamiento y por tanto, dura lo que dure la vida de cada sujeto. (Ruz, 2003)

Por otro lado, Descartes, cuando instauró su frase "pienso y luego existo", dio el paso para que la filosofía dejara de ser doctrina del ser (metafísica), y se constituya en la doctrina de pensar. Antes el ser condicionaba el pensamiento, ahora es el pensamiento quien condiciona el ser. (Ruz, 2003) 
Si bien, Descartes no escribió obras pedagógicas, sus afanes por descubrir un procedimiento de investigación, tuvieron en la enseñanza grandes influencias. Una de ellas es, el discurso del método, del que desprenden muchas reglas para la investigación. La principal es: presentar a los alumnos ideas claras. Hacerlos amar la claridad y la precisión, infundiéndoles aversión por todo lo que es indeterminado y obscuro.

Además, el racionalismo, sentó bases como que, todas las verdades deben derivar siempre de un principio evidente por sí mismo. Y esta actitud, significó desterrar conocimientos erróneos o inadecuados, no solo los que provienen de la experiencia de los sentidos (ya que me pueden engañar), sino también de todo principio establecido antes de la investigación, como sucede por ejemplo en la historia, en la moral, etc. (Zuretti, Hustoria de la Educación, 1959)

En la vereda opuesta al racionalismo, el empirista Hume y su crítica al razonamiento inductivo, nos deja como herencia un mundo regido por la probabilidad, una realidad a la que tenemos que incorporar la incertidumbre como uno de sus componentes esenciales. La ciencia, en consecuencia, estará construida en función de "conjeturas y refutaciones" y ya no volverá a ser ese conocimiento cierto, objetivo, y eterno al que se aspiró en la modernidad. (Ordosgoitti, 2016)

Siguiendo las ideas empiristas, el sociólogo Emile Durkheim, desarrolla un pensamiento que va desde la teoría y la práctica docente, y aborda el fenómeno educativo anclado a la sociología, (será la sociedad la que considere a los individuos para la construcción del saber).

En este sentido la sociología de la educación toma relevancia para la educación, en vista de que los saberes se convendrán impartir y facilitar a los estudiantes de acuerdo al contexto donde se desarrolla el estudiante, y procurar realizarlos con aprendizajes significativos y funcionales frente a realidades distintas de los educandos, funda así un nuevo estilo de aprendizaje adaptado las realidades y necesidades sociales.

Es decir, que Durkheim percibe a la educación como un elemento social, en cuyos procesos de enseñanza-aprendizaje, los docentes necesariamente deberán tener en cuenta, la configuración del educando, y sus particularidades, esto transforma la educación, ya que se convierte en un proceso más subjetivo e integral para los estudiantes.

En otro orden de ideas, el naturalista, Rousseau, en su teoría desarrolla la idea del maestro como amigo educativo, que acompaña y corrige desde la buena fe, deja de lado el papel de autoridad, para convertirse en un ser más enfático en lograr al estudiante virtuoso. Porque, como Rousseau considera que el hombre es bueno por naturaleza, y que la sociedad la que lo corrompe, su objetivo es lograr un sujeto que, alienado de la sociedad, logra ser virtuosa, y puede desde el compañerismo actuar de guía, con los demás. 
Partiendo de esa base, Pestalozzi, cree que la educación natural, es la correcta. Considera que el proceso educativo debe construirse desde los sentimientos del amor, la confianza, la gratitud, la obediencia, la conciencia del deber y del derecho. (Paniagua, 2015)

El método de Pestalozzi, fue admirado por muchos, entre ellos, Herbart, quien empezó su formación pedagógica siguiendo sus pasos, como se adelantó en el apartado II, creía que la manera de lograr el aprendizaje, era a través de la instrucción, que la instrucción no debía ser un juego, sino que necesitaba generar interés genuino, por ello ingenió cinco pasos para la enseñanza. Pero además le prestó especial atención a la disciplina, como los niños son "ligeros e irreflexivos", necesitan límites que impidan que se dañen a sí mismos. (Una idea completamente opuesta a la de Rousseau)

La disciplina implica órdenes para dirigir la actividad, prohibiciones para impedir manifestaciones peligrosas y vigilancia para asegurar el cumplimiento de unas y otras. La acción disciplinaria debe tener en cuenta las disposiciones naturales y el género de vida del educando. Sus disposiciones pueden impulsar o contener, ser permanentes o temporarias. (Zuretti, Breve Historia de la Educación, 1998)

Mientras que el sociólogo más destacado, Karl Marx, como ya se anticipó en el capítulo II, divide la sociedad en dos clases sociales, los burgueses y los proletarios, siendo los primeros quienes tienen capital, y los segundos quienes prestan su fuerza de trabajo en favor de los burgueses. Esa fuerza de trabajo, que genera producción, otorga al producto un valor, pero el salario que los trabajadores perciben no se asemeja al valor que si trabajo tiene, y esa plusvalía es la que genera la riqueza de los burgueses.

Para revertir esa situación, Marx propone, que los proletarios, deberían realizar una revolución, una lucha, para obtener parte del capital y así lograr una sociedad comunista, de iguales. Previo a la revolución, los proletarios trabajadores, deberían despertar de su alienación, deberían comprender el valor de su trabajo.

Éste filósofo y sociólogo, entiende que la alienación de la clase trabajadora proviene de la educación que reciben, que es manipulada e ideologizada por los grupos de poder. Es por ello, que para alcanzar ese despertar, esa revalorización de su trabajo y eliminar el adiestramiento, piensa en la educación de la colectividad, así toman importancia a las tareas pedagógicas, a las bibliotecas, y la investigación. ${ }^{5}$ (Paniagua, 2015)

\section{Enseñanza Jurídica}

La enseñanza jurídica, es de gran importancia, dado que, si entendemos al derecho como herramienta de cambio, comprenderemos que el uso de esa herramienta estará en manos de los profesionales del derecho. Entonces, será la formación de cada uno de ellos, la que guíe sus actos en el ejercicio de la profesión, y las consecuencias sociales que estos actos impliquen.

\footnotetext{
${ }^{5}$ Manifiesto Comunista, 1848 (Karl Marx; Frederic Engels)
} 
La enseñanza jurídica actual, se caracteriza, por no generar herramientas críticas en los educandos, es decir, el resultado es la poca permeabilidad para incorporar contenidos que tiendan a desafiar o no reproducir el discurso jurídico dominante, entendido como positivismo jurídico. (Mariana Manzo - Carolina Monte, 2018).

Puede que la razón por la que los profesionales del derecho, no tengan herramientas críticas, sea porque el Derecho no puede abordarse a través de una sola escuela o teoría, que solucione todos los problemas que plantea el Derecho. Las teorías son muchas y cambian, los que subsisten son los problemas referidos al Derecho.

De esta forma la enseñanza de cada pensamiento puede brindar herramientas para que el alumno construya su propio concepto de Derecho. (Manzo, 2008)

Mientras que muchas, sino son todas las Facultades de Derecho, dividen las materias en cátedras de pensamientos o ideologías distintas, y solo algunas de aquellas cátedras enseñan el pensamiento opuesto al que pertenecen. Éste es quizás, un primer punto que dificulta el pensamiento crítico en los estudiantes.

Esto significa, que son pocos los conocimientos ciertos y objetivos, está incorporada la incertidumbre, tal como pretendía el empirista David Hume. (Ordosgoitti, 2016)

Otro punto, que influye en el desarrollo o no del pensamiento crítico, son los métodos utilizados a la hora de plantear problemas del Derecho. Entre los mencionados métodos, se encuentran el método del caso y el método del caso - problema.

El primero, consiste en plantear un determinado caso, que tiene una solución determinada, por lo que la tarea de los alumnos consistirá en el uso de la lógica deductiva, ya que tendrán que descubrir cuál es la norma aplicable al caso. Mientras que el método del caso - problema, implica plantear un caso con soluciones diversas, que implicará para los y las estudiantes, la tarea de encontrar algunas de las soluciones normativas, (en el sentido amplio de la norma), aplicable al caso y a partir de allí, elegir la que consideren más conveniente y efectiva. Todo lo cual deberá ser fundado. ( Carlos Lista- Ana María Brigido y Silvana Begala, 2007)

Además, no será igual, el hecho de que un profesor/a empiece la clase diciendo, por ejemplo: - "El Derecho, es un conjunto de normas y principios, tendientes a alcanzar un fin inmediato..." // O "El proceso, es una serie gradual progresiva y concatenada de actos jurídicos, que tienen por fin lograr un acto judicial efectivo...”. A que, en cambio, se empiece la clase a través de una pregunta, ¿Qué creen que es el Derecho? (Manzo, 2008)

En síntesis, existen diversos puntos que se pueden considerar, para pensar en que es lo que genera la poca capacidad crítica en los y las estudiantes de Derecho, como ser la relación entre profesores/as, y alumnos/as, las estructuras dentro de un aula, etc. (Mariana Manzo - Carolina Monte, 2018)

Siguiendo a los racionalistas, Platón, por ejemplo, hablaba en "La alegoría de las cavernas", de que una los sujetos que se encontraban encerradas y encadenadas, se movió 
y sintió las cadenas, y solo así pudo liberarse, salir y entender a través de la razón, en donde se encontraba antes y en donde se encontraba ahora, que eran las figuras que veía desde el interior, y demás. Pero ¿cómo lograr ese despertar, que con posterioridad será orientado por la razón? ¿Qué provocó que ese sujeto se moviera?, ¿La metafórica incomodidad? Si es así, el hecho de que uno de los sujetos se sintiera incómoda y eligiera moverse, esto le permitiera razonar y comprender una realidad distinta a la que había vivido toda su vida, dependería más bien de su personalidad.

Por lo tanto, no dependería de la forma de enseñar el Derecho, sino de características personales de los y las estudiantes, que luego podrán ser acompañadas con el uso de la razón. Es decir, que ninguno de los puntos considerados con anterioridad imposibilitaría de manera determinante el desarrollo del pensamiento crítico en el alumnado.

Aristóteles, coincide con su maestro al considerar que el Estado tiene un rol social importante frente a la educación, y en considerar la capacidad de razonar como algo esencial en el aprendizaje, pero completa el pensamiento, por cuanto añade que, el aprendizaje es un proceso, dentro del cual influye la experiencia y que no sería posible, sin la vida en comunidad regulada por el Estado. (Larecio, 2010)

Terminando con el abordaje de los autores racionalistas, Descartes decía, que pensamos y luego existimos o, dicho de otra forma, existimos porque pensamos, y por ende somos quienes somos porque lo pensamos. (Zuretti, Breve Historia de la Educación, 1998)

Me recuerda, a una pregunta, que hacen algunos profesores en sus primeras clases, “PPor qué eligieron abogacía?”. En la pedagogía hay quien dice, que las preguntas como, “QQué es el derecho?”, tienen por fin despertar la curiosidad a través de la duda, esto significa, que se pretende con ellas que los/as alumnos/as, se den cuenta de que no saben y se interesen por saber. (Paniagua, 2015) Recordemos que, (y me permito saltar del racionalismo al constructivismo), era Herbart quien hablaba de lograr primero el interés de los educandos para luego instruirlos.

Pero la pregunta ¿Por qué estudias abogacía?, pareciera ser distinta. Hablar de porqué estudiamos abogacía, implica hablar de cuáles son los intereses de el/la estudiante, cuál fue el camino recorrido hasta hacer esa elección, que o quienes influyeron en esa elección, y por ende traer al aula, el contexto de quienes se encuentran en ella. Dando cuenta, que tal como pensaba el otro autor constructivista abordado, Pestalozzi, la educación se debe lograr, atravesando el contexto social del educando (Campos-Badilla, 2016)

Durkheim, empirista, también tiene en consideración el contexto social a la hora de educar, y es aún más claro en esta idea. Habla de educar de acuerdo al contexto de cada estudiante, y de aprendizaje funcional a las realidades de aquellos. Razón por la cual, serán los docentes los encargados de indagar y lo harán a través de las mismas preguntas mencionadas. Así el proceso educativo, se convertirá en un proceso más subjetivo para cada estudiante. 
También contribuyen a la subjetivación del proceso educativo, las ideas de Rousseau, como bien ya se dijo, este autor naturalista, confía en la naturaleza humana, y plantea una idea de profesor, alejado de la autoridad, y abordando la corrección y guía de buena fe. Propone una solución para uno de los puntos planteados, la relación profesor-alumno.

En Derecho esta relación, no es tan distinta a las demás, pero es particular si tenemos en cuenta, por ejemplo, que, en los finales, aunque no haya exigencia formal, existen juicios sociales, que implican una cierta vestimenta, un cierto vocabulario, y otras cuestiones simbólicas como ser que el asiento del profesor se encuentre a una mayor altura. Todo esto desde una perspectiva sociológica asevera, la autoridad del profesor frente a los educandos. (Mariana Manzo - Carolina Monte, 2018).

Ahora bien, para dar respuesta a los puntos planteados, lo primero es plantearlos cuestionarlos, y es precisamente por esto que Marx es uno de los autores más destacados en la historia. Porque fue él quien entendió, que, para lograr un cambio social, es indispensable salir de la alienación, se relaciona directamente con la educación que se recibe.

En otras palabras, resulta indispensable evitar la monopolización del pensamiento. Se deben presentar, las distintas posturas del derecho, para que los educandos decidan, con cual se identifican, sólo así se lograría, el cese de predominio del positivismo jurídico, un verdadero cambio en los profesionales de Derecho, su influencia en la sociedad. Y por ende un cambio social.

\section{Conclusiones}

Para arribar a la conclusión, voy a transcribir un fragmento de una entrevista al Dr. Miguel Ángel Ciuro Caldani del año 2018 en la UBA, cuando presentaba la Maestría de Filosofía del Derecho.

\subsection{Trialismo jurídico}

A lo largo de este trabajo, se habló sobre distintas perspectivas, sobre los sujetos, sobre la educación, sobre la educación jurídica y sobre el Derecho.

Para Hans Kelsen el derecho se había introducido en temas psicológicos, biológicos, morales y hasta teológicos, y poniendo en peligro la verdadera ciencia jurídica.

Por ello, Kelsen emprendió "la tarea de elaborar una teoría depurada de toda ideología política y de todo elemento de la ciencia de la naturaleza, y consciente de tener un objeto regido por leyes que le son propias" (La Teoría pura del Derecho). Trato de encontrar un objeto de estudio que fuera propio de la materia, que alcanzara un pensamiento claro e intenta probar que sólo existe el derecho positivo concebido como una serie de normas coordinadas y sistematizadas. Pretende dar a la teoría del derecho la objetividad, entiende al derecho como una ciencia cuyo único objeto es el derecho. Y en 
conjunto con esta teoría que el positivismo jurídico cobra tanta fuerza y se mantiene a la fecha.

Como una postura superadora, en 1958, Werner Goldschmidt propone el trialismo jurídico.

Las corrientes actuales que disuelven el pensamiento jurídico se inscribe en una fuerte referencia a la vida. La vida puede ser algo muy ambiguo, pero estamos condenados a las ambigüedades, ¿irían a un médico que no esté interesado por la vida?

Al Derecho le debe importar la vida, el trialismo se ocupa de la vida, y se ocupa de intereses, nos mueven los intereses, por eso el derecho debe ocuparse de ellos, nos movemos utilizando fuerzas y el derecho debe ocuparse de ello.

El trialismo jurídico, como su palabra lo indica, tiene como base tres divisiones, (sociológica, normológica, y axiológica o valorativa).

Se necesitan diálogos entre economía, religión, lengua, arte, hay una cantidad de disciplinas de los que la dimensión sociológica puede tomar otras disciplinas, sin disolverse. Lo hace a través de las adjudicaciones de potencia.

La división normológica capta esa realidad social, y la dimensión axiológica lo hace a través de una valoración. Es decir, hay cuestiones que son comunes al derecho, luego, hay especificidades temporales, espaciales, y personales.

No se ha tratado suficientemente las especificidades personales, porque el derecho es en cada persona, no es una abstracción generalizada, sino que cada persona tiene una conjunción jurídica. Por ejemplo, hay quienes creen que el Derecho de propiedad es un gran título, porque tienen muchas propiedades, el derecho penal puede verse como derecho de protección cuando se es víctima, otros pueden verlo como sanción que le atribuye la sociedad, es decir tiene rasgos comunes y específicos.

En la segunda mitad del siglo XX, hemos aprendido a ver otras cosas, entendimos que además del mercado y del capitalismo, hay democracia, derechos humanos, hemos aprendido a ver la dignidad del ser humano, y por eso hay que reconstruir las ramas del derecho agregando otras, que sean lecturas transversales, que no nieguen las ramas tradicionales pero que vean la presencia de problemas humanos y no puede tratarse en abstracto.

Por ejemplo: La quiebra de un hospital no puede ser solo un objeto de derecho de quiebra porque está el derecho a la salud, el problema de salud no se resuelve en lo administrativo, o civil, lo que dice es que esas ramas tienen que dialogar. Lo mismo con la educación, no podría cerrarse una escuela sin tener en cuenta al educando y al educador y su derecho superior, tiene que tenerse en cuenta el principio de protección del educando.

Estamos preocupados por equilibrar la economía, pero para equilibrar la economía necesitamos la democracia, y para que la democracia equilibre la economía se necesita la filosofía del derecho. (Caldani, 2018) 


\subsection{Abordaje personal}

En conclusión, ninguna de las teorías abordadas, es correcta, ni incorrecta, tampoco son completas y tampoco son opuestas cómo puede presentarse. Se trata de teorías complementarias.

Para lograr una educación efectiva, para lograr el interés de los educandos, y por ende para que se sientan impulsados en lograr una educación completa, no pueden dejar de lado sus características personales.

Abordar la enseñanza, desde las características personales de cada alumno o alumna, despertaría un interés diferenciado.

Si a su vez, a ese interés diferenciado, le sumamos el presentar para cada materia distintas perspectivas, evitaríamos incomodidades de los educandos, y tendríamos la posibilidad de que elaboren un concepto personal sobre el derecho, y las muy diversas materias en las que el derecho influye.

Es un hecho, que las personas si bien tenemos características comunes, también las tenemos muy distintas, que cada quien logre una concepción personal de derecho sería entonces, que existan concepciones de derecho con características comunes, pero muy distintas entre sí y muy enriquecedor en el mundo jurídico.

A su vez, abordar la enseñanza de una manera más subjetiva, podría resultar favorable a la vocación. Hoy por hoy, es muy conocida la frase "serás lo que debas ser o serás abogado”. Por tanto, estimular la formación de profesionales con vocación, podría significar una re-valorización de la profesión. Y de los futuros profesionales. A su vez, el campo laboral del derecho es tan amplio, que la enseñanza jurídica que sirva de guía, en cuanto de qué forma se quiere abordar la profesión, podría evitar el ejercicio frustrado de la abogacía.

Ahora bien, si presentamos solamente un discurso o una forma de pensamiento del derecho, la enseñanza del derecho resultaría hegemónica, y ese único discurso presentado, resultaría una forma de Poder, omitiendo la existencia del derecho como una herramienta de cambio.

La presentación del derecho, desde sus distintas perspectivas, como lo pretende el trialismo jurídico, favorecería al pensamiento crítico y esto permitiría a los abogados/as, el presentarse como actores en las disputas sobre el capital jurídico. No se pretende negar la importancia del positivismo jurídico, abordado en el trialismo como el aspecto normológico, sino que lo se pretende es ampliarlo a través de su complemento con los aspectos sociológico, y valorativo.

Tener en cuenta, los distintos pensamientos, presentados en relación a la pedagogía, como complementarios, (racionalismo; empirismo; constructivismo; naturalismo y materialismo histórico), trasladarlos al campo jurídico, a través del método trialista, permitiría dar paso al pensamiento crítico. 
El pensamiento crítico, es de tal importancia porque lograría que el derecho y las normas, dejen de ser estáticos, empiecen a ser dinámicos, en el sentido de que se adapten a la realidad social en que transitan. Respondan a las necesidades sociales contemporáneas, y evitaría el intentar adaptar dichas necesidades al monopolio social. (Lista, 2014)

Por ejemplo: Actualmente, en la Provincia de Jujuy, estamos pasando por una emergencia en cuestiones de género, de acuerdo al observatorio de "Ni una menos Jujuy" somos la provincia con mayor tasa de femicidios en Argentina.

Los juzgados especializados en género, declaran su incompetencia en la mayoría de las denuncias presentadas. Basándose, exclusivamente en cuestiones formales y olvidando que el objetivo de la Ley de creación, es hacer frente a la necesidad de proteger a las mujeres de los actos de violencia.

Es decir, que existe una dificultad de la judicatura para fundamentar sus decisiones en cuestiones que no sean normológicas. En la enseñanza jurídica en general, no se instruye al pensamiento, a través de la experiencia, la razón, las características personales, la buena fé del educador, como la harían cada uno de los autores planteados. Es decir, los educadores en general, no tienen o no transmiten los pensamientos pedagógicos abordados. Por ende, se sostiene una forma de enseñanza hegemónica, que es el positivismo jurídico, razón por la cual los diversos profesionales, no tienen las herramientas para abordar otras formas jurídicas de pensamiento que no sea basado en normas. Y por tanto, difícilmente puedan resolver cuestiones, que poco tienen que ver con los formalismos jurídicos.

Si a esto le sumamos, un Poder Legislativo, con igual formación, el resultado puede ser catastrófico, ya que el órgano creador de leyes, reproduciría distintas formas del derecho ya existente, no podría innovar verdaderamente y por tanto, los demás profesionales del derecho, estarán limitados a ese derecho estático.

Todo esto, implica abordar de manera ineficiente, problemáticas, actuales, dinámicas y evolutivas, con un derecho desactualizado, incompleto, estático y por lo tanto ineficiente.

En síntesis, para lograr el ejercicio social eficiente de la abogacía, se requiere el pensamiento crítico en los profesionales, para lo cual es necesaria la transformación de la pedagogía jurídica, a través de un cambio en la formación docente por medio del uso complementario de las distintas formas de pensamiento pedagógico, con la ayuda del trialismo jurídico.

\section{Referencias bibliográficas}

Carlos Lista- Ana María Brigido y Silvana Begala. (2007). La enseñanza jurídica: tendencias y desafíos. 8 Congreso Nacional de Sociología Jurídica: derecho, democracia y sociedad. 
Biswas, N. (2007). Knowledge and pedagogy. En Biswas, Technological pedagogical knowledge.

Caldani, M. A. (18 de Octubre de 2018).

https://www.youtube.com/watch?v=bRwGscW4dsc. (UBA, Entrevistador, \& UBA, Editor) Buenos Aires, Argentina. Obtenido de https://www.youtube.com/watch?v=bRwGscW4dsc

Campero, M. B. (2017). Descartes y la contrucción del sujeto. Revista FACTÓTUM. Campos-Badilla, X. (2016). Influencia de los postuladosde Jean Jacques Rousseau,Johan Heinrich Pestalozzi,Antonio Rosmini-Serbati,Johann Friedrich Herbart yKarl Marx sobre las Teorías de la Educación de Siglos XX y XXI. Acta Academica.

Funes, E. (2004). Subjetividad y sociedad en la teoría de Emilio Durkheim. sociales uba. Obtenido de http://www.sociales.uba.ar/wp-content/uploads/20-FunesDurkheim.pdf

Gadotti, M. (2003). HISTORIA DE LAS IDEAS PEDAGÓGICAS. Sao Pablo: Editorial Siglo XXI.

Larecio, D. (2010). Vidas, opiniones y sentencias de los filósofos más ilustres". Traducidas del griego por José Ortiz y Sanz. para la biblioteca virtual universal. Biblioteca Virtual Universal

Lista, C. (16 de Mayo de 2014). Educación y profesión jurídica. (R. d. Humanidades., Entrevistador) youtube. Cordoba. Obtenido de https://www.youtube.com/watch?v=ojDyjcP8ETA

Manzo, M. (2008). La influencia de la educación jurídica en la formación valorativa de los abogados. Revista Academia.

Mariana Manzo - Carolina Monte. (2018). Enseñanza del derecho, currículum oculto y feminismo. . Crítica y Resistencias. Revista de conflictos sociales latinoamericanos.

Montero, S. A. (2012). Causalidad y Sujeto: el problema del empirismo de Hume. Recuperado a partir de http://revistas.uptc.edu.co/index.php/cuestiones_filosofia/article/view/615.

Ordosgoitti, E. A. (2016). El Sujeto y las Ideas en la teoría del conocimiento de Hume. Asociación Centro de Investigaciones Socioculturales de Venezuela (CISCUVE). Obtenido de www.ciscuve.org https://ciscuve.org/?p=

Paniagua, R. A. (2015). Teoría de Educación y Filosofía. Revista Ensayos Pedagogicos.

Ruz, I. (2003). La educación según Aristóteles. Madrid. URL La Educación Según Aristóteles (slideshare.net)

Zuretti, J. C. (1959). Hustoria de la Educación. Editorial Itinerarium - Colección Escuela. Zuretti, J. C. (1998). Breve Historia de la Educación. Editorial Claridad - Colección Escuela. 